Review

\title{
Probiotics for the Primary and Secondary Prevention of C. difficile Infections: A Meta-analysis and Systematic Review
}

\author{
Lynne V. McFarland \\ Department of Medicinal Chemistry, University of Washington, VA Puget Sound Healthcare System, \\ 1660 S. Columbian Way, S-152, Seattle, WA 98108, USA; E-Mail: 1vmcfarl@u.washington.edu; \\ Tel.: +1-206-277-1780; Fax: +1-206-764-2935
}

Academic Editor: Yung-Fu Chang

Received: 9 March 2015 / Accepted: 2 April 2015 / Published: 13 April 2015

\begin{abstract}
Clostridium difficile infections are a global clinical concern and are one of the leading causes of nosocomial outbreaks. Preventing these infections has benefited from multidisciplinary infection control strategies and new antibiotics, but the problem persists. Probiotics are effective in preventing antibiotic-associated diarrhea and may also be a beneficial strategy for $C$. difficile infections, but randomized controlled trials are scarce. This meta-analysis pools 21 randomized, controlled trials for primary prevention of $C$. difficile infections (CDI) and four trials for secondary prevention of $C$. difficile recurrences and assesses the efficacy of specific probiotic strains. Four probiotics significantly improved primary CDI prevention: (Saccharomyces boulardii, Lactobacillus casei DN114001, a mixture of L. acidophilus and Bifidobacterium bifidum, and a mixture of L. acidophilus, L. casei and L. rhamnosus). None of the tested probiotics significantly improved secondary prevention of CDI. More confirmatory randomized trials are needed to establish if probiotics are useful for preventing $C$. difficile infections.
\end{abstract}

Keywords: probiotics; clostridium difficile infections; diarrhea; meta-analysis

\section{Introduction}

Clostridium difficile infections (CDI) have been a difficult clinical issue for over four decades, with a nearly one-half a million cases per year in the U.S., resulting in 29,000 deaths per year, increased costs of healthcare, outbreaks of CDI in hospitals and long-term care facilities and 83,000 cases of recurrent 
CDI per year the U.S. [1]. Prevention of CDI has relied on multidisciplinary infection control practices, but guidelines have been found to be difficult to implement globally [2,3].

An innovative strategy to prevent CDI involves using probiotics at the same time antibiotics are given. One recent quasi-experimental study was done in Canada, which gave the mixture of L. acidophilus, L. casei and L. rhamnosus $(\mathrm{BioK}+)$ to all patients receiving antibiotics at two hospitals over time and found a significant reduction in the incidence of CDI cases and recurrences at these facilities [4]. Some probiotic strains have been found to be effective for prevention of antibiotic-associated diarrhea (AAD) and for the treatment of CDI [5]. Since CDI accounts for nearly one-third of all AAD cases, this strategy is worth evaluating since CDI persists in impacting our healthcare systems. However, studies of CDI prevention and probiotics have been largely limited to CDI being evaluated as a secondary outcome of AAD studies, leading to underpowerment for CDI outcomes [6]. The technique of meta-analysis allows the pooling of different trials to overcome the low power bias due to the small individual sample sizes. In this paper, randomized, controlled trials of good quality will be pooled to assess probiotic strains for primary and secondary prevention of CDI.

\section{Results}

\subsection{Initial Screening of Data Search}

The literature review yielded 474 abstracts relating to probiotics and CDI that were screened for inclusion. Of those, 323 were excluded after initial screening according to our exclusion criteria (Figure 1): reviews $(n=152)$, pre-clinical animal models or phase two studies for pharmacokinetics, formulation or safety $(n=81)$, no control group or case series $(n=54)$, commentaries $(n=20)$ or not randomized $(n=16)$.

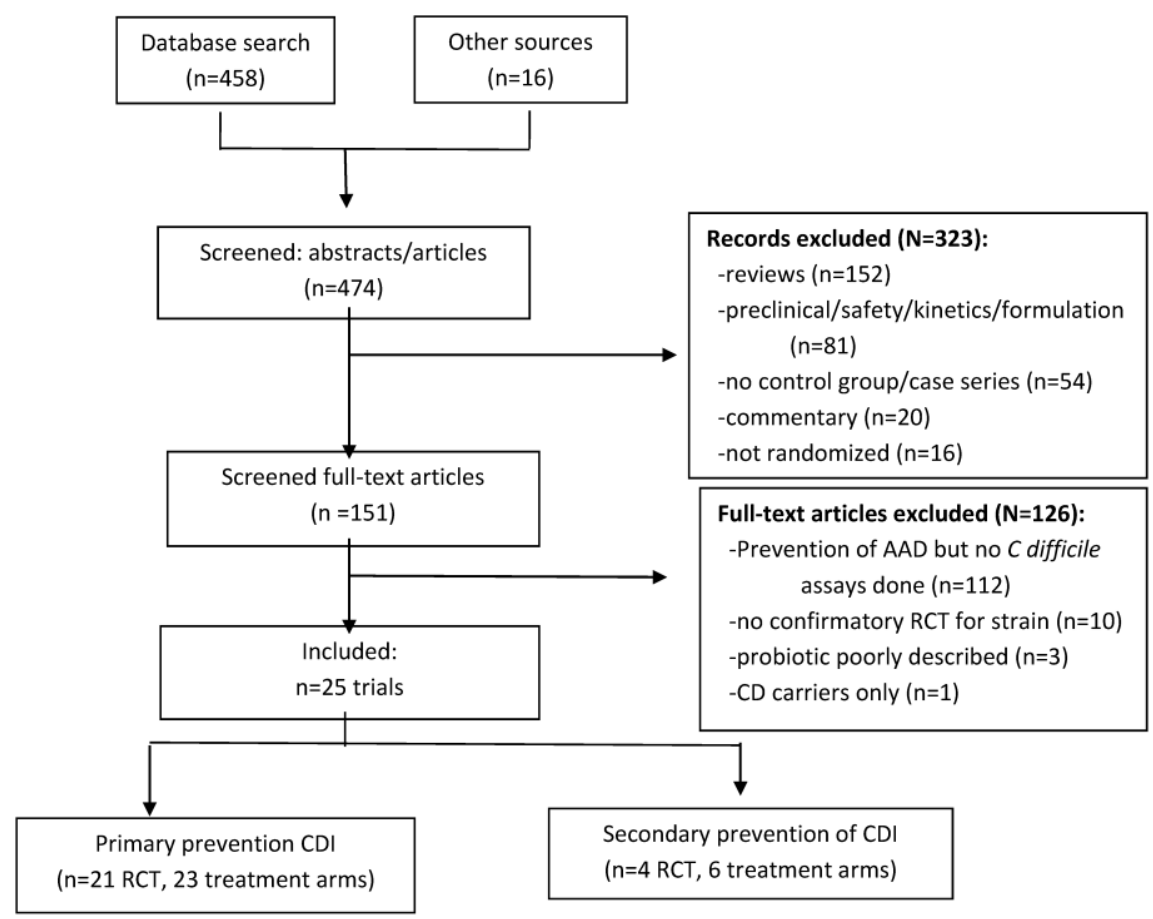

Figure 1. PRISMA flow-diagram of literature search of probiotics for primary or secondary prevention of Clostridium difficile infections (CDI). 


\subsection{Secondary Screening of Full Articles}

Of 151 full-text articles or meeting abstracts screened, 126 were excluded. Most $(n=112)$ were trials for the prevention of $\mathrm{AAD}$ that lacked any $C$. difficile outcomes, or the outcome was only for asymptomatic carriage of $C$. difficile $(n=1)$ or the probiotic genus and species were not provided $(n=3)$. Probiotic interventions lacking confirmatory trials (that is, only one trial/probiotic type) were excluded $(n=10)$, as shown in Table $1[7-16]$.

\subsection{Included Trials}

For primary CDI prevention, 21 trials (23 treatment arms) were included [17-37] and for secondary CDI prevention, four trials (six treatment arms) were included [38-41]. Of the 25 randomized controlled trials included, three had multiple treatment arms, [28,37,39] resulting in a total of 29 treatment arms, totaling 4476 participants. Most articles are full-text, peer-reviewed articles ( $n=23,92 \%)$, but two are available only as meeting abstracts [28,33]. The sample sizes of the trials ranged from 42 to 437 , with a mean number per trial of $83 \pm 52$ in probiotic groups and $82 \pm 51$ in control groups $(p=0.89)$. Two articles were translated from their original languages into English: one in Hebrew [34] and one in Spanish [23]. The 25 trials were carried out 11 countries: USA (9, 36\%), Canada (3, 12\%), U.K. (3, 12\%), China $(2,8 \%)$, Turkey $(2,8 \%)$ and one trial $(4 \%)$ in each: Chile, Finland, Germany, Israel, Italy and Poland. Of the 25 trials included, 19 (76\%) tested a single strain of probiotic and six (24\%) tested a mixture of probiotic strains. Of the 21 primary prevention trials, the primary outcome of the trials was typically the prevention of $\operatorname{AAD}(16,76 \%)$, while three $(14 \%)$ designated the prevention of CDI as a secondary outcome [28,32,34], and two trials $(10 \%)$ designated CDI as an adverse event associated with their primary outcomes (either prevention of ventilator-associated pneumonia [29] or Helicobacter pylori eradication therapy [20]. All four of the secondary CDI prevention trials had the prevention of CDI recurrences as their primary outcome [38-41].

\subsection{Study Design}

Degree of blinding in primary prevention. Of the 21 trials, most $(n=18,86 \%)$ were double-blinded (used placebos that were of identical appearance as the probiotic formulation), while three (14\%) had open controls, which used either no treatments [20,25] or had an active control with another strain (L. casei Shirota) of probiotic as a comparator [31].

Degree of blinding in secondary prevention. All four trials had double-blinded, placebo controlled controls.

Attrition in primary prevention trials. As shown in Table 2, attrition ranged from $0 \%-43 \%$ in the 21 trials, drop-outs typically due to adverse events or loss to follow-up. Six trials (29\%) reported no attrition, eight (38\%) had low attrition frequencies from $1 \%-10 \%$, four $(19 \%)$ had moderate attrition from $12 \%-26 \%$, while three (14\%) reported high attrition frequencies $(38 \%-43 \%)$. Of the 14 trials with attrition, only two (14\%) used Intent-to-Treat (ITT) analysis [20,23], while most (86\%) used as-perprotocol (APP) analysis.

Attrition in secondary prevention trials. Of the four trials, three reported attrition rates from no attrition [39,41], to $16 \%$ attrition [38], but attrition was not reported in one trial [40]. 
Table 1. Prevention of Clostridium difficile infections (CDI) for trials with only one study for specific probiotic type.

\begin{tabular}{|c|c|c|c|c|c|c|c|}
\hline Probiotic & $\begin{array}{c}\text { Eligible Antibiotic } \\
\text { Exposures } \\
\end{array}$ & $\begin{array}{c}\text { Daily Dose of } \\
\text { Probiotic (cfu/day) }\end{array}$ & $\begin{array}{c}\text { Duration of Probiotic } \\
\text { Treatment } \\
\end{array}$ & $\begin{array}{l}\text { Duration } \\
\text { Follow-up }\end{array}$ & $\begin{array}{c}\text { CDI in Probiotic } \\
\text { Group (\%) } \\
\end{array}$ & $\begin{array}{c}\text { CDI in Control } \\
\text { Group (\%) } \\
\end{array}$ & Reference \\
\hline \multicolumn{8}{|c|}{ Primary prevention of CDI } \\
\hline L. casei Shirota & $\mathrm{nr}$ & $6.5 \times 10^{9}$ & duration +1 week & 4 weeks & $9 / 76(0 \%) \mathrm{ns}$ & $1 / 82(1.2 \%)$ & Wong 2014 [7] \\
\hline L. acidophilus & mixed, $77 \%$ beta-lactams & $6 \times 10^{10}$ & 2 weeks & 0 & $0 / 23(0 \%) \mathrm{ns}$ & $1 / 16(6.2 \%)$ & Safdar 2008 [8] \\
\hline L. plantarum $299 v$ & mixed & $1 \times 10^{10}$ & duration +1 week & 1 week & $1 / 74(1.3 \%) \mathrm{ns}$ & $0 / 76(0 \%)$ & Lonnermark 2010 [9] \\
\hline Bacillus clausii & mixed, beta-lactams & $4 \times 10^{9}$ & duration & 6 weeks & $0 / 162(0 \%) \mathrm{ns}$ & $1 / 160(0.6 \%)$ & Destura 2008 [10] \\
\hline C. butyricum 588 & mixed, $87 \%$ beta-lactams & $1-4 \times 10^{7}$ & 6 days & 0 & $0 / 83(0 \%) \mathrm{ns}$ & $0 / 27(0 \%)$ & Seki 2003 [11] \\
\hline L rhamnosus (strains E/N, Oxy, Pen) & mixed, mostly pen and ceph & $4 \times 10^{10}$ & duration $(\mathrm{x}=8$ day) & 2 weeks & $3 / 120(2.5 \%) \mathrm{ns}$ & $7 / 120(5.8 \%)$ & Ruszczynski 2008 [12] \\
\hline L. rhamnosus $\mathrm{GG}+$ L. acido. La5 + Bifido. lactis $\mathrm{Bb} 12$ & mixed, nr types & $5 \times 10^{10}$ & 2 weeks & 0 & $0 / 34(0 \%) \mathrm{ns}$ & $1 / 29(3.4 \%)$ & Wenus 2008 [13] \\
\hline $\begin{array}{l}\text { L. acidophilus (CUL } 60 \text { and CUL 21) + Bifido. } \\
\text { bifidum CUL20 + Bifido. lactis CUL34 }\end{array}$ & mixed, $21 \%$ single, $70 \%$ pen & $6 \times 10^{10}$ & 3 weeks & 10 weeks & $12 / 1470(0.8 \%) \mathrm{ns}$ & $17 / 1471(1.2 \%)$ & Allen 2013 [14] \\
\hline VSL\#3 & mixed, $75 \%$ pen & $9 \times 10^{11}$ & duration +1 week & 3 weeks & $0 / 117(0 \%) \mathrm{ns}$ & $0 / 112(0 \%)$ & Selinger 2013 [15] \\
\hline \multicolumn{8}{|c|}{ Secondary prevention of CDI } \\
\hline L. plantarum $299 \mathrm{v}$ & mixed & $5 \times 10^{10}$ & 5.4 weeks & 4.5 weeks & $\begin{array}{c}4 / 11(36 \%) \\
\text { recurred }\end{array}$ & 6/9 (67\%) & Wullt 2003 [16] \\
\hline
\end{tabular}

Abbreviations: Bifido., Bifidobacterium; C., Clostridium; cfu, colony-forming unit; L., Lactobacillus; ns, not significant; VSL\#3, contains Bifido. breve, Bifido. longum,

Bifido. infantis, L. acidophilus, L. plantarum, L. casei, L. bulgaricus, Strept. thermophilus; x, mean. 
Table 2. Study design description for primary prevention of $C$. difficile infections from studies of Probiotics for the Prevention of Antibiotic-associated diarrhea.

\begin{tabular}{|c|c|c|c|c|c|}
\hline Enrolled population & $\%$ Attrition & $\begin{array}{c}\text { Single or Multiple Types of } \\
\text { Inciting Antibiotics } \\
\end{array}$ & $\begin{array}{c}\text { Most Common Type } \\
\text { of Antibiotic }\end{array}$ & Type(s) of Infections & Reference \\
\hline adults, I & 43 & $59 \%$ multiple & $36 \%$ cepha & mixed, $\mathrm{nr}$ & Surawicz 1989 [17] \\
\hline adults, I & 38 & $82 \%$ multiple & beta-lactams & mixed, $\mathrm{nr}$ & McFarland 1995 [18] \\
\hline elderly, I & 4.2 & $\mathrm{nr}$ & $\mathrm{nr}$ & $\mathrm{nr}$ & Lewis 1998 [19] \\
\hline adult, $\mathrm{O}$ & 3.3 & $100 \%$ multiple & amox and clarithromycin & H. pylori infections & Duman 2005 [20] \\
\hline pediatric, $\mathrm{I} \& \mathrm{O}$ & 8.5 & $\mathrm{nr}$ & $41 \%$ cepha & $68 \%$ resp, $29 \%$ otitis media & Kotowska 2005 [21] \\
\hline adults, I & 0 & $\mathrm{nr}$ & $83 \%$ beta-lactams & $\mathrm{nr}$ & Can 2006 [22] \\
\hline adults, $\mathrm{O}$ & 4.6 & $100 \%$ single & $100 \%$ amox & $88 \%$ resp & Bravo 2008 [23] \\
\hline adults, I & 26 & $69 \%$ single & mixed, nr & $\mathrm{nr}$ & Pozzoni 2012 [24] \\
\hline pediatric, I & 15 & $\mathrm{nr}$ & $52 \%$ cepha & resp & Shan 2014 [25] \\
\hline pediatric, $\mathrm{O}$ & 28.7 & $\mathrm{nr}$ & $66 \%$ amox & $74 \%$ otitis media, $26 \%$ resp & Arvola 1999 [26] \\
\hline adults, I & 11.6 & $\mathrm{nr}$ & $69 \%$ beta-lactams & $\mathrm{nr}$ & Thomas 2001 [27] \\
\hline adults, I & 0 & $\mathrm{nr}$ & cepha & $\mathrm{nr}$ & Miller 2008a [28] \\
\hline adults, I & 0 & $69 \%$ single & $50 \%$ cepha & $\mathrm{nr}$ & Miller 2008b [28] \\
\hline adults, I & 5.5 & only $34 \%$ with VAP on abx & $\mathrm{nr}$ & pneumonia & Morrow 2010 [29] \\
\hline adults, I & 19 & $61 \%$ single & $66 \%$ amox or cepha & $49 \%$ resp & Hickson 2007 [30] \\
\hline adults, I & 0 & $\mathrm{nr}$ & $60 \%$ amp or cepha & $80 \%$ resp or GU & Dietrich 2014 [31] \\
\hline elderly, I & 8 & $\mathrm{nr}$ & $\mathrm{nr}$ & $\mathrm{nr}$ & Plummer 2004 [32] \\
\hline adults, I & 0 & $\mathrm{nr}$ & mixed & $\mathrm{nr}$ & Rafiq 2007 [33] \\
\hline adults, I & 0 & $\mathrm{nr}$ & $48 \%$ ceph & $\mathrm{nr}$ & Stein 2007 [34] \\
\hline adults, I & 0 & $\mathrm{nr}$ & $59 \%$ quinolones & $92 \%$ resp & Beausoleil 2007 [35] \\
\hline adults, I\&O & 7.4 & $\mathrm{nr}$ & $78 \%$ beta-lactams & $39 \%$ resp & Sampalis 2010 [36] \\
\hline adults, I & 9 & $\mathrm{nr}$ & $41 \%$ cepha & $47 \%$ resp & Gao 2010a [37] \\
\hline adults, I & 7 & $\mathrm{nr}$ & $37 \%$ cepha & $47 \%$ resp & Gao 2010b [37] \\
\hline
\end{tabular}

Abbreviations: amox, amoxicillin; amp, ampicillin; cepha, cephalosporin; GU, genital-urinary infections; I, inpatient; nr, not reported; O, outpatient; resp, respiratory infections; VAP, ventilator-associated pneumonia. 


\subsection{Patient Population}

Primary prevention trials. Most of the 21 trials (15, 71\%) were done at one hospital, while six (29\%) were done at multiple sites (hospitals and/or clinics) [18,20,21,30,32,36]. Most ( $n=16,76 \%$ ) enrolled inpatients, three (14\%) of the trials enrolled only outpatients and two $(9 \%)$ had a mixture of inpatients and outpatients. As shown in Table 2, most of the 21 trials enrolled adult participants $(n=18,86 \%)$ and three $(14 \%)$ enrolled children [21,25,26], and all trials included both genders. Race or ethnicity was not reported in most clinical trials.

Secondary prevention trials. Most of the four trials were done at multiple sites: three sites [38,41] or four sites [39], while one trial was done at one site [40]. Three trials enrolled both inpatients and outpatients [38-40], but one trial did not report the type of patient enrolled [41]. All four trials enrolled only adult patients. Two trials enrolled only patients with recurrent CDI [39,41], while two enrolled patients with either incident or recurrent CDI $[38,40]$.

\subsection{Antibiotic Exposure}

Primary prevention trials. As shown in Table 2, the types of antibiotic exposures varied widely from single antibiotics to multiple types. Of the 21 trials, only seven reported if single or multiple antibiotics were prescribed, most $(88 \%)$ had a mix of single and multiple antibiotics. One trial enrolled patients with only amoxicillin use [23]. The most common types of antibiotic exposure were beta-lactams including penicillins and cephalosporins. Of the 21 trials, 11 (52\%) reported the type of infection requiring antibiotics, which was most commonly for respiratory infections.

Secondary prevention trials. Of the four trials, only one reported the types of inciting antibiotics, but none reported the original disease indication for the antibiotics. In this one trial, $31 \%$ were single antibiotics and 69\% were multiple types, with the most common type being cephalosporins [38].

\subsection{Interventions}

Probiotics in primary CDI prevention trials. Details of the intervention for the $21 \mathrm{RCT}$ (23 treatment arms) for the primary prevention of CDI are given in Table 3. Five different types of probiotics were described in the 21 trials: three single-strain probiotics (Saccharomyces boulardii CNCM I-745 (S. boulardii), Lactobacillus rhamnosus GG, L. casei DN114001)) and two types of probiotic mixtures: (L. acidophilus and Bifidobacterium bifidum) and (L. acidophilus CL1285 and L. casei LBC80R and L. rhamnosus CLR2 ( $\mathrm{La}+\mathrm{Lc}+\mathrm{Lr})$ ). Newer strain designations for several probiotics and the retrospective review of older studies may have used different strain designations, but were, in fact, the same strain. The most recent strain designations are used in this study. The most current strain designation for S. boulardii is CNCM I-745, the registration number at the Pasteur Institute [42], but older studies also refer to this strain as $S$. boulardii lyo, or $S$. boulardii, with no strain designation or by the brand name "Florastor". L. casei DN1 14001 is also cited as the brand name "Actimel". The mixture of L. acidophilus CL1285 and L. casei LBC80R and L. rhamnosus CLR2 is also cited as the brand name "Bio K+" [43]. 
Table 3. Characteristics of probiotic and control treatments and rate of $C$. difficile infections (CDI) by group.

\begin{tabular}{|c|c|c|c|c|c|c|c|c|}
\hline Probiotic & Daily Dose (cfu/d) & Formulation & Duration Treatment & Follow-up (weeks) & CDI in Probiotic & CDI in Controls & Power & Reference \\
\hline S. boulardii & $2 \times 10^{10}$ & capsules & duration +2 weeks & 0 & $3(2.6 \%)$ & $5(7.8 \%)$ & $26.5 \%$ & Surawicz 1989 [17] \\
\hline S. boulardii & $3 \times 10^{10}$ & capsules & duration +3 days & 7 & $3(3.1 \%)$ & $4(4.2 \%)$ & $2.6 \%$ & McFarland 1995 [18] \\
\hline S. boulardii & $4.5 \times 10^{9}$ & capsules & duration $(\mathrm{x}=7$ days $)$ & 0 & $5(15 \%)$ & $3(8.3 \%)$ & $7.2 \%$ & Lewis 1998 [19] \\
\hline S. boulardii & $1 \times 10^{10}$ & capsules & duration $(\mathrm{x}=2$ weeks $)$ & 4 days & $0(0 \%)$ & $1(0.5 \%)$ & $3.3 \%$ & Duman 2005 [20] \\
\hline S. boulardii & $1 \times 10^{10}$ & wafers & duration ( $\mathrm{x}=1$ week) & 0 & $3(2.5 \%)$ & $10(7.9 \%)$ & $35.6 \%$ & Kotowska 2005 [21] \\
\hline S. boulardii & $1 \times 10^{10}$ & capsules & duration & 4 & $0(0 \%)$ & $2(2.6 \%)$ & $9.1 \%$ & Can 2006 [22] \\
\hline S. boulardii & $1 \times 10^{10}$ & capsules & 12 days & 9 days & $0(0 \%)$ & $0(0 \%)$ & -- & Bravo 2008 [23] \\
\hline S. boulardii & $1 \times 10^{10}$ & capsules & duration +7 days & 12 & $3(2.8 \%)$ & $2(2 \%)$ & $3 \%$ & Pozzoni 2012 [24] \\
\hline S. boulardii & $1 \times 10^{10}$ & powder & duration $(\mathrm{x}=2$ weeks $)$ & 2 & $1(0.7 \%)$ & $8(5.6 \%)$ & $51.9 \%$ & Shan 2014 [25] \\
\hline L. rhamnosus GG & $4 \times 10^{10}$ & capsules & duration $(\mathrm{x}=7-10$ day $)$ & 12 & $1(1.6 \%)$ & $1(1.7 \%)$ & $10 \%$ & Arvola 1999 [26] \\
\hline L. rhamnosus GG & $2 \times 10^{10}$ & capsules & 2 weeks & 1 & $2(1.5 \%)$ & $3(2.2 \%)$ & $2.7 \%$ & Thomas 2001 [27] \\
\hline L. rhamnosus GG & $4 \times 10^{10}$ & capsules & duration $(\mathrm{x}=2$ weeks $)$ & 4 & $4(4.2 \%)$ & $7(7.4 \%)$ & $9.2 \%$ & Miller 2008a [28] \\
\hline L. rhamnosus GG & $1.2 \times 10^{11}$ & capsules & duration $(\mathrm{x}=2$ weeks $)$ & 4 & $2(1.3 \%)$ & 0 & $11.2 \%$ & Miller 2008b [28] \\
\hline L. rhamnosus GG & $4 \times 10^{9}$ & capsules & duration $(x=15$ day $)$ & 0 & $4(5.8 \%)$ & $13(18.6 \%)$ & $52.9 \%$ & Morrow 2010 [29] \\
\hline L. casei DN 114001 & $2 \times 10^{10}$ & drink & duration +1 week & 4 & $0(0 \%)$ & $9(17 \%)$ & $81 \%$ & Hickson 2007 [30] \\
\hline L. casei DN 114001 & $2 \times 10^{10}$ & drink & duration $(\mathrm{x}=6$ days $)$ & 0 & $0(0 \%)$ & $3(10 \%)$ & $21.3 \%$ & Dietrich 2014 [31] \\
\hline L acidophilus + Bifido. bifidum & $2 \times 10^{10}$ & capsules & $20 \mathrm{~d}$ & 0 & $2(2.9 \%)$ & $5(7.2 \%)$ & $11.5 \%$ & Plummer 2004 [32] \\
\hline L acidophilus + Bifido. bifidum & cfu nr (3g/day) & capsules & duration or LOS & 0 & $5(11 \%)$ & $22(40 \%)$ & $88.0 \%$ & Rafiq 2007 [33] \\
\hline L acidophilus + Bifido. bifidum & $6 \times 10^{9}$ & capsules & 3 weeks & 0 & $3(14.3 \%)$ & $1(4.8 \%)$ & $7.2 \%$ & Stein 2007 [34] \\
\hline $\begin{array}{l}\text { L. acidophilus CL1285 + L. casei } \\
\text { LBC80R + L. rhamnosus CLR2 }\end{array}$ & $5 \times 10^{10}$ & milk & duration $(\mathrm{x}=7-8$ day $)$ & 3 & $1(2.3 \%)$ & $7(15.6 \%)$ & $44.2 \%$ & Beausoleil 2007 [35] \\
\hline $\begin{array}{l}\text { L. acidophilus CL1285 + L. casei } \\
\text { LBC80R + L. rhamnosus CLR2 }\end{array}$ & $5 \times 10^{10}$ & milk & duration +5 days & 3 & $1(0.5 \%)$ & $4(1.8 \%)$ & $12.5 \%$ & Sampalis 2010 [36] \\
\hline $\begin{array}{l}\text { L. acidophilus CL1285 + L. casei } \\
\text { LBC80R + L. rhamnosus CLR2 }\end{array}$ & $5 \times 10^{10}$ & capsules & duration +5 days & 3 & $8(9.4 \%)$ & $20(23.8 \%)$ & $64 \%$ & Gao 2010a [37] \\
\hline $\begin{array}{l}\text { L. acidophilus CL1285 + L. casei } \\
\text { LBC80R + L. rhamnosus CLR2 }\end{array}$ & $1 \times 10^{11}$ & capsules & duration +5 days & 3 & $1(1.2 \%)$ & $20(23.8 \%)$ & $99.2 \%$ & Gao 2010b [37] \\
\hline
\end{tabular}

Abbreviation: Bifido., Bifidobacterium; CDI, C. difficile infections; cfu, colony-forming units; L., Lactobacillus; LOS, length of stay; nr, not reported; S., Saccharomyces; x, mean. 
The daily dose of probiotics varied widely from a lower daily dose in three treatment arms $\left(4-6 \times 10^{9}\right)[19,29,34]$ to higher doses ranging from $1-12 \times 10^{10}$ colony-forming units (cfu) per day, while one study did not report their daily dose by cfu/d [33].

Most of the 23 treatment arms used a capsule formulation (74\%), while four (17\%) were given in milk or other drinks, or as powder $(4 \%)$ or in wafers $(4 \%)$.

Probiotics were given in conjunction with the antibiotics (usually started within $48-72 \mathrm{~h}$ of the antibiotic) and continued for either the duration of the antibiotic (12 treatment arms, 52\%) or continued for 3-14 days after antibiotics were discontinued ( 7 arms, 30\%). Four treatment arms gave the probiotic for a prescribed period (ranging from 14-21 days), regardless of the duration of antibiotics [23,27,32,34].

The duration of follow-up post-antibiotic and probiotic intervention ranged from 0-90 days. Eight (35\%) of the treatment arms did not follow patients after the intervention had been discontinued. Most trial arms followed patients for 2-4 weeks (9 arms, 39\%), or 1 week ( $2 \mathrm{arms}, 9 \%$ ) or for only four days (1, arm, 4\%), while three (13\%) had prolonged follow-up periods from seven to 12 weeks [18,24,26].

As CDI was usually a secondary outcome, not all enrolled trial participants were assayed for C. difficile, most trials tested for $C$. difficile when participants developed diarrheal symptoms, but not all trials successfully assayed all participants with diarrhea, nor provided data on the number of participants tested for $C$. difficile. One trial planned a priori to assay for $C$. difficile at enrollment, at the end of the intervention and end of follow-up, and successfully assessed 133 (69\%) of trial participants, regardless of diarrheal symptoms [18]. Only three other trials reported the frequency of testing for C. difficile (done only if diarrhea developed), which was in a limited number of participants: $n=16$ [20] or $n=46$ [36], but one study only tested 50\% (4/8) participants with diarrhea [23].

Probiotics in secondary CDI prevention trials. As shown in Table 4, four of six treatment arms tested a single strain of yeast ( $S$. boulardii) $[38,39]$ and two treatment arms tested a single strain of bacteria (L. rhamnosus $G G$ ) [40,41]. The three treatment arms in one trial combined $S$. boulardii or placebo in three separate antibiotic adjunctive treatments [either low dose vancomycin (500 mg/day), high dose vancomycin (2 g/day) or metronidazole (1 g/day)] [39]. The doses of vancomycin or metronidazole adjuncts were not controlled in the other three trials and were under the discretion of the patient's primary provider. The daily dose of the probiotic varied from $2-3 \times 10^{10}$ day [38,39] to $3 \times 10^{11}$ [41], but daily dose was not provided in one trial [40]. Five of the treatment arms had a capsule formulation, while one used a probiotic yogurt [40]. The duration of probiotic intervention varied from 3-4 weeks, except in one trial that gave the intervention during adjunctive antibiotic therapy (typically 10-14 days), then extended the intervention for another three weeks [41]. The duration of follow-up was usually four weeks post-intervention, except for one trial that followed patients for 8.6 weeks [41]. 
Table 4. Secondary prevention by probiotic type for treatment of Clostridium difficile infections (CDI).

\begin{tabular}{|c|c|c|c|c|c|c|c|c|c|c|}
\hline $\begin{array}{l}\text { History } \\
\text { of CDI }\end{array}$ & Pop-ulation & Type of controls & $\begin{array}{l}\text { Adjunctive therapy } \\
\text { (daily dose) }\end{array}$ & Probiotic & $\begin{array}{l}\text { Probiotic } \\
\text { daily dose } \\
\text { (cfu/day) }\end{array}$ & $\begin{array}{l}\text { Duration treated } \\
\text { (follow-up) }\end{array}$ & $\begin{array}{c}\text { Frequency CDI } \\
\text { recurrences in } \\
\text { probiotic } \\
\end{array}$ & $\begin{array}{c}\text { Frequency CDI } \\
\text { recurrences in controls }\end{array}$ & Power (\%) & Reference \\
\hline $\mathrm{I} / \mathrm{R}$ & $\begin{array}{l}124 \text { adults, } \\
\text { In \& Out }\end{array}$ & placebo & V or M (varied) & S. boulardii & $3 \times 10^{10}$ & 4 weeks (4 weeks) & $15 / 57(26.3 \%)^{*}$ & $30 / 67(44.8 \%)$ & 49.5 & McFarland 1994 [38] \\
\hline $\mathrm{R}$ & $\begin{array}{l}83 \text { adults, } \\
\text { In \& Out }\end{array}$ & placebo & $\mathrm{V}(500 \mathrm{mg})$ & S. boulardii & $2 \times 10^{10}$ & 4 weeks (4 weeks) & $23 / 45(51 \%)$ & $17 / 38(44.7 \%)$ & 5.3 & Surawicz 2000a [39] \\
\hline $\mathrm{R}$ & $\begin{array}{l}32 \text { adults, } \\
\text { In \& Out }\end{array}$ & placebo & $\mathrm{V}(2 \mathrm{~g})$ & S. boulardii & $2 \times 10^{10}$ & 4 weeks (4 weeks) & $3 / 18(17 \%)^{*}$ & $7 / 14(50 \%)$ & 35.9 & Surawicz 2000b [39] \\
\hline $\mathrm{R}$ & $\begin{array}{l}53 \text { adults, } \\
\text { In \& Out }\end{array}$ & placebo & M (1g) & S. boulardii & $2 \times 10^{10}$ & 4 weeks (4 weeks) & $13 / 27(48 \%)$ & $13 / 26(50 \%)$ & 3.3 & Surawicz 2000c [39] \\
\hline $\mathrm{I} / \mathrm{R}$ & $\begin{array}{l}25 \text { adults, } \\
\text { In \& Out }\end{array}$ & placebo & $\mathrm{V}(\mathrm{nr}) \mathrm{M}(\mathrm{nr})$ & $\begin{array}{l}\text { L rhamnosus } \\
\text { GG }\end{array}$ & $\mathrm{nr}$ & 3 weeks (4 weeks) & $4 / 11(36.4 \%)$ & $5 / 14(35.7 \%)$ & 5.7 & Pochapin 2000 [40] \\
\hline $\mathrm{R}$ & 15 adults & placebo & $\begin{array}{l}20 \% \mathrm{~V}(\mathrm{nr}) \\
80 \% \mathrm{M}(\mathrm{nr})\end{array}$ & $\begin{array}{l}\text { L rhamnosus } \\
\text { GG + inulin }\end{array}$ & $3 \times 10^{11}$ & $\begin{array}{c}\text { duration } a b x+21 \\
\text { days (8.6) }\end{array}$ & $3 / 8(37.5 \%)$ & $1 / 7(14.3 \%)$ & 5.3 & Lawrence 2005 [41] \\
\hline
\end{tabular}

$* p<0.05$, Abbreviations: abx, antibiotics; CDI, Clostridium difficile infection; I, initial CDI episode; In, inpatient; L., Lactobacillus; M, metronidazole; Md, median; $\mathrm{nr}$, not reported in paper/abstract; Out, outpatient; R, recurrent CDI; S., Saccharomyces; V, vancomycin. 


\subsection{Pooled Efficacy of Probiotics for Primary CDI Prevention}

Meta-analysis. A meta-analysis of the 23 treatment arms of probiotic versus controls was performed and the pooled results indicated a low degree of heterogeneity $\left(\mathrm{I}^{2}=17.2 \%, p=0.23\right)$, so a fixed-effect model was used. As shown by the forest plot in Figure 2, when trials were pooled by similar types of probiotic species, four of five types of tested probiotic types were significantly effective for primary CDI prevention: S. boulardii (pRR = 0.50, 95\% C.I. 0.29, 0.85), L. casei DN114001 (pRR=0.07, 95\% C.I. 0.01, 0.55), the mixture of L. acidophilus and Bifido. bifidum ( $\mathrm{pRR}=0.41,95 \%$ C.I. 0.21, 0.80), and the mixture of $L$. acidophilus and $L$. casei and L. rhamnosus (pRR $=0.21,95 \%$ C.I. $0.11,0.40)$. The pooled results for L. rhamnosus GG did not reach statistical significance. A funnel plot (data not shown) and Egger's text for publication bias did not show significant publication bias $(p=0.17)$.

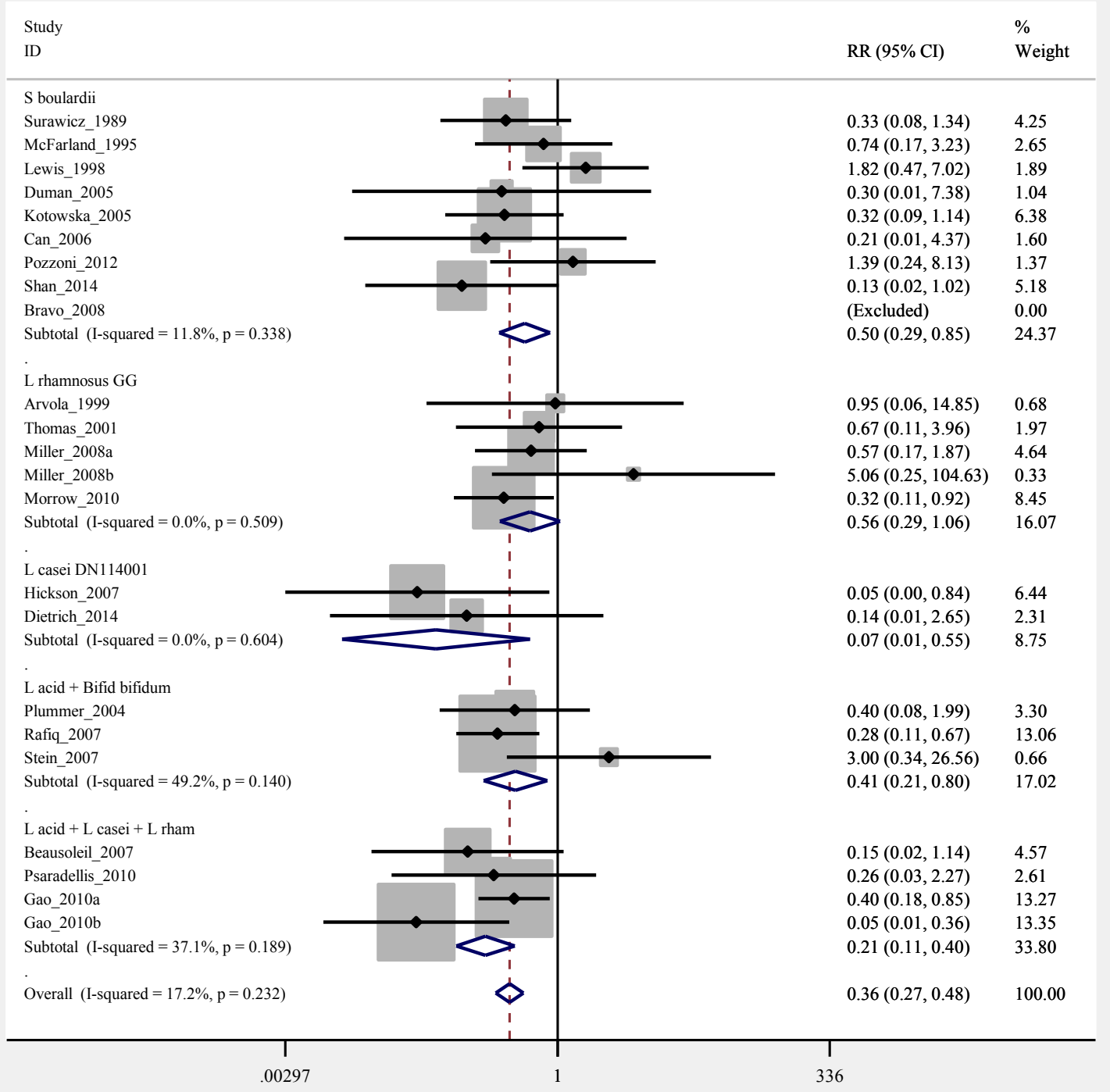

Figure 2. Forest plot of fixed effects model of meta-analysis of primary prevention of C. difficile disease by sub-group of probiotic type, $\mathrm{x}$-axis indicates relative risk. 
Sub-group analysis. Results from the meta-regression analysis for the adjunctive use of probiotics primary prevention of CDI did not find significant differences in associations between the study population (adult versus pediatric, $p=0.68)$, or daily dose of probiotic $\left(\geq 10^{10} \mathrm{cfu} /\right.$ day versus $<10^{10} \mathrm{cfu} /$ day, $p=0.18$ ). Only the probiotic strain group showed significance, confirming the validity of analyzing efficacy by strain type.

\subsection{Pooled Efficacy of Probiotics for Secondary CDI Prevention}

Meta-analysis. A meta-analysis of the six treatment arms of probiotic versus controls was performed and the pooled results indicated a moderate degree of heterogeneity $\left(\mathrm{I}^{2}=35.4 \%, p=0.17\right)$, so a fixed-effect model was used. As shown by the forest plot in Figure 3, when trials were pooled by similar types of probiotic species, neither $S$. boulardii nor L. rhamnosus GG was significantly efficious for secondary CDI prevention. Publication bias was not assessed due to the limited number of available trials.

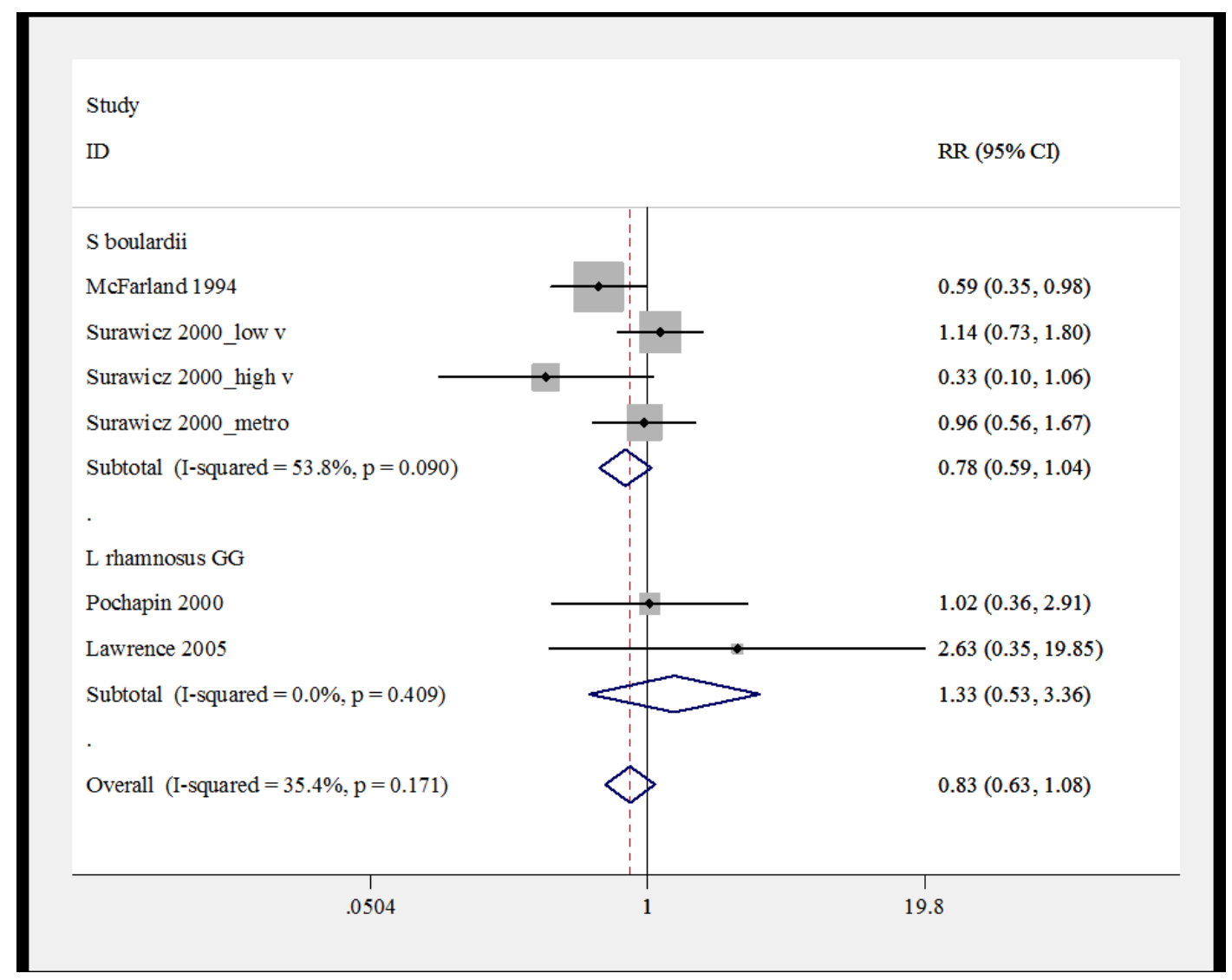

Figure 3. Forest plot of fixed effects model of meta-analysis of secondary prevention of C. difficile disease by sub-group of probiotic type, $\mathrm{x}$-axis indicates relative risk.

\section{Discussion}

Clinical recommendations for the use of probiotics in CDI disease has been limited by the scarcity of well-done, randomized controlled trials using CDI as their powered, primary outcome. Most of the evidence results from prevention of AAD trials, which include CDI only as a secondary outcome and did not consider this outcome when calculating the needed study size for their trials $(52 \%$ had $\leq 10 \%$ power). As a consequence, most individual trials have not found statistically significant efficacy for 
probiotics and the prevention of CDI. This meta-analysis pooled the results of these trials, resulting in a significant increase in power and detected some (but not all) probiotic types were effective in preventing primary cases of CDI. The evidence for probiotics and the secondary prevention of CDI recurrences remains hampered by a lack of randomized, controlled trials.

As research on probiotics has evolved, the efficacy and mechanisms-of-action has been found to be highly strain-specific, requiring that dissimilar types of probiotics to be analyzed as separate subgroups [44]. Previous meta-analyses on probiotics for the prevention of CDI done before these guidelines were established pooled dissimilar types of probiotic species in their analysis $[5,45]$. A recent metaanalysis chose to pool their main outcomes across probiotic species, based on the hypothesis that the efficacy should be similar, as the mechanisms-of-action is similar for different probiotics [46]. I would disagree with this hypothesis, as different probiotic strains can have different mechanisms-of-action and resulting degrees of efficacies [47]. Another recent meta-analysis did not separate the different types of probiotics in their nine included trials [48]. More recent meta-analyses have presented their results by probiotic sub-groups, but were not as comprehensive as this meta-analysis: One meta-analysis included 11 trials [6] and another was only done in five pediatric trials [49]. Another meta-analysis included 20 trials and did present pooled results by sub-groups, but the data was not presented within specific pooled probiotic groups [50].

The strengths of this meta-analysis include the extensive literature search of both established literature databases, use of grey literature and correspondence with experts in the field, inclusion of a large number of high to moderate quality randomized, controlled clinical trials, the analysis of the efficacy for both primary CDI prevention and secondary CDI prevention by probiotic type sub-groups and the use of standardized methods adhering to current meta-analytic guidelines. The result is a comprehensive evaluation of the types of probiotics that are effective in preventing CDI, allowing clinicians to evaluate whether the use of probiotics may be effective in their patients. Limitations of this meta-analysis are inherent in the reporting of published trials with missing data (for example, not all reported the types of antibiotics or the number of participants tested for $C$. difficile) and the limited number of confirmatory trials tested for each type of probiotic. Of the 15 different types of probiotics with randomized trials for the prevention of CDI, only five (33\%) had multiple trials, allowing pooling of their results. More well-done trials need to be done testing the same types of probiotics.

\section{Methods}

\subsection{Aims}

The two aims of this review were to assess the use of specific probiotics for: (1) primary prevention of $C$. difficile disease (CDI) and (2) secondary prevention of $C$. difficile recurrences. Primary prevention of CDI is defined as people without diarrhea symptoms who are exposed to antibiotics and are given the intervention who do not develop diarrhea associated with a positive $C$. difficile assay (culture, immune assay, cytotoxin test or other assay) within two months exposure to the inciting antibiotic. Secondary prevention of CDI (prevention of CDI recurrences) is defined as people who have recovered from at least one prior episode of CDI, are asymptomatic (no diarrhea) at the time of the intervention and do not develop a recurrence of CDI within 1-2 months of follow-up. 


\subsection{Search Strategy}

This meta-analysis followed PRISMA (Preferred Reporting Items for Systematic reviews and Meta-Analysis) statement guidelines [51] and guidelines using clearly delineated parameters, a priori inclusion and exclusion criteria and standardized data extraction tools [52,53] Systematic searches of PubMed (1960-2015), EMBASE (1974-2015), Cochrane Database of Systematic Reviews (1990-2015), ISI Web of Science (2000-2015) and three on-line clinical trial registries: Cochrane Central Register of Controlled trials (http://www.cochrane.org), MetaRegister of Controlled Trials (http:www.controlled-trials.com/mrct) and National Institutes of Health (http://www.clinicaltrials.gov) were done. All bibliographies from relevant studies were used to do a recursive search. Additional sources included: extensive grey literature search including abstracts from annual infectious disease and gastroenterology meetings, probiotic product websites, communication with experts in the field and published authors. Search terms included: C. difficile prevention, antibiotic-associated diarrhea, randomized controlled trials and specific probiotic strains. Search strategies were broad-based initially, then narrowed to the disease and population of interest. Abstracts of all citations and retrieved studies were reviewed and rated for inclusion. Full articles were retrieved if probiotics were given prevent diarrhea or treat $C$. difficile infections.

\subsection{Inclusion and Exclusion Criteria}

Inclusion criteria included randomized (well described or partially) controlled trials (RCT), blinded or open trials, in pediatric or adult populations (inpatient or outpatients), published in peer-reviewed journals or on clinical trial websites, or as meeting abstracts. Non-English language trials were translated and included whenever possible. Exclusion criteria included pre-clinical studies, safety, kinetic or formulation phase 2 studies, case reports or case series, duplicate reports, trials of unspecified types of probiotics, non-randomized trials, incomplete or no outcomes reported, or if translation could not be obtained. Probiotic strains with only one randomized controlled trial (lacking at least one other confirmatory trial) were also excluded.

\subsection{Data Extraction}

The data was extracted from a database from a previous meta-analysis on primary prevention and updated with recent publications, while secondary prevention articles were added [6]. For articles published in abstract form only or for any missing significant data in full articles, further information was sought by contacting authors or by the company manufacturing the probiotic product. Using a standardized data extraction form, the following data was systematically collected: authors, year of publication and journal, population data (age range, setting, types of antibiotic exposures, types of inciting diseases), study aims and outcomes, study methods (study design, eligibility criteria, sample size calculations, interim analysis, statistical methods used, recruitment methods, subgroup analysis done), randomization (method of randomization allocation, randomization method), degree of blinding (open, single or double), intervention data (probiotic strains used, daily dose, duration of treatment, duration of follow-up, type of control used, treatment concealment), types of $C$. difficile assays done, results (balanced randomization achieved, attrition rate and reasons, comparison of treatment groups by 
demographics, etc., CONSORT flow-chart provided), outcome data [by group, intent-to-treat (ITT) or as-per-protocol (APP) analysis], safety data (adverse events reported by group), discussion points (limitations, generalizability and comparison of study results to published papers), clinical trial registration, location of protocol, and source of funding.

\subsection{Interventions}

Included trials had participants who were randomized to either a probiotic group or a control group. The type of control group may have included either a placebo (blinded study) or no treatment (open study). The type of probiotic intervention included probiotics in any formulation (e.g., capsule, sachet, tablets, drink, etc.). Trials investigating non-specific probiotics or yogurts (e.g., articles not providing the probiotic strain(s) used) were excluded. The most recent probiotic strain designations are presented in this study for those strains whose names have changed over time (older articles may have reported a different strain designation). The taxonomy of the probiotic strain type was confirmed by correspondence with authors or the manufacturing companies.

\subsection{Statistical Analysis}

Statistical analysis was performed using Stata software version 12 (Stata Corporation, College Station, Texas) to calculate pooled relative risks (pRR), bias estimates and number-needed-to-treat statistics. Univariate analysis results were analyzed using $X^{2}$ test or Fisher's exact test for small cell sizes $(<5)$ with a significance level of $p<0.05$. Meta-analysis was conducted for primary outcomes (CDI) using models to calculate the pooled relative risk and corresponding $95 \%$ confidence interval $(95 \% \mathrm{CI})$ using the DerSimonian Laird method. Heterogeneity across trials was evaluated using Cochran Q test based on pooled relative risks by the Mantel-Haenazel method [54]. If the studies were homogenous, a fixed effects model was used; if studies were heterogeneous, a random effect model was employed. A $p$-value $<0.05$ is considered statistically significant. The models used in this analysis were weighted by sample size, as study quality did not improve the fit.

If significant heterogeneity was found, subgroup analyses were conducted to determine the potential sources of heterogeneity. To explore possible explanations for heterogeneity, a priori subgroup analyses were conducted on study population (adult versus pediatric) and daily dose $\left(\geq 1 \times 10^{10}\right.$ colony-forming units (cfu) per day or $<1 \times 10^{10} \mathrm{cfu} /$ day). A meta-regression was done without the subgroup indicator and compared to a model with the subgroup indicator included. The difference in $\mathrm{tau}^{2}$ estimates from the two models indicates the proportion of study heterogeneity explained by the subgroup covariate (between study variance).

\subsection{Publication Bias}

To assess for publication bias, a funnel plot, as well as a weighted regression (Egger's test) and a rank correlation test (Begg's test for small study effects) were conducted [52,55]. Funnel plots show graphically that as sample sizes of trials increase, the precision is estimating the underlying treatment effect increases, which results in the effect estimates (relative risks) from small trials scattering more widely at the bottom of the graph and narrower scattering among larger studies. In the absence of 
publication bias, the funnel plot resembles a symmetrical inverted funnel. Reporting bias (smaller studies showing no protective effect) often are not published, and are indicated by an asymmetrical appearance with a gap in the bottom left of a funnel plot [56].

\section{Conclusions}

Four different types of probiotics were found to be effective for primary prevention of CDI (S. boulardii, L. casei DN114001, the mixture of L. acidophilus and Bifido. bifidum and the mixture of L. acidophilus, L. casei and L. rhamnosus). L. rhamnosus GG was not significantly efficious for the primary prevention of CDI and the other 10 types of probiotics lacked a second trial, so pooling of their outcomes was not possible. More clinical experience with these four probiotics might be recommended to confirm if they are effective in larger populations of patients.

Only two types of probiotics ( $S$. boulardii and L. rhamnosus GG) had sufficient numbers of trials for to assess secondary prevention of CDI by meta-analysis, but none of the pooled results reached statistical significance. It may be that neither of these strains were effective in this analysis for preventing CDI recurrences, but based on prior experience and use of these probiotics (mechanism of action studies, case series, etc.), there are indications that these probiotic strains may be effective if an effective combination of probiotic and anti-C. difficile antibiotics can be determined [57,58].

\section{Acknowledgements}

This study was unfunded.

\section{Conflicts of Interest}

Lynne McFarland has received fees as a speaker (Biocodex, France and Lallemand, France) and is on the scientific advisory board of BioK+, Canada.

\section{References}

1. Lessa, F.C.; Mu, Y.; Winston, L.G.; Dumyati, G.K.; Farley, M.M.; Beldavs, Z.G.; Kast, K.; Holzbauer, S.M.; Meek, J.I.; Cohen, J.; et al. Burden of Clostridium difficile infection in the United States. N. Engl. J. Med. 2015, 372, 825-834.

2. Surawicz, C.M.; Brandt, L.J.; Binion, D.G.; Ananthakrishnan, A.N.; Curry, S.R.; Gilligan, P.H.; McFarland, L.V.; Mellow, M.; Zuckerbraun, B.S. Guidelines for diagnosis, treatment, and prevention of Clostridium difficile infections. Am. J. Gastroenterol. 2013, 108, 478-498.

3. Martin, M.; Zingg, W.; Knoll, E.; Wilson, C.; Dettenkofer, M. National European guidelines for the prevention of Clostridium difficile infection: A systematic qualitative review. J. Hosp. Infect. 2014, 87, 212-219.

4. Maziade, P.J.; Andriessen, J.A.; Pereira, P.; Currie, B.; Goldstein, E.J.C. Impact of adding prophylactic probiotics to a bundle of standard preventative measures for Clostridium difficile infections: Enhanced and sustained decrease in the incidence and severity of infection at a community hospital. Curr. Med. Res. Opin. 2013, 29, 1341-1347. 
5. McFarland, L.V. Meta-analysis of probiotics for the prevention of antibiotic associated diarrhea and the treatment of Clostridium difficile disease. Am. J. Gastroenterol. 2006, 101, 812-822.

6. Johnson, S.; Maziade, P.J.; McFarland, L.V.; Trick, W.; Donskey, C.; Currie, B.; Low, D.E.; Goldstein, E.J. Is primary prevention of Clostridium difficile infection possible with specific probiotics? Int. J. Infect. Dis. 2012, 16, e786-e792.

7. Wong, S.; Jamous, A.; O’Driscoll, J.; Sekhar, R.; Weldon, M.; Yau, C.Y.; Hirani, S.P.; Grimble, G.; Forbes, A. A Lactobacillus casei Shirota probiotic drink reduces antibiotic-associated diarrhoea in patients with spinal cord injuries: A randomised controlled trial. Br. J. Nutr. 2014, 111, 672-678.

8. Safdar, N.; Barigala, R.; Said, A.; McKinley, L. Feasibility and tolerability of probiotics for prevention of antibiotic-associated diarrhea in hospitalized US military veterans. J. Clin. Pharm. Ther. 2008, 33, 663-668.

9. Lönnermark, E.; Friman, V.; Lappas, G.; Sandberg, T.; Berggren, A.; Adlerberth, I. Intake of Lactobacillus plantarum reduces certain gastrointestinal symptoms during treatment with antibiotics. J. Clin. Gastroenterol. 2010, 44, 106-112.

10. Destura, R.V. Bacillus clausii in preventing antibiotic-associated diarrhea among Filipino infants and children: A multi-center, randomized, open-label clinical trial of efficacy and safety. Available online: http://en.sanofi.com/img/content/study/ENTER_L_01125_summary.pdf (accessed on 3 June 2013).

11. Seki, H.; Shiohara, M.; Matsumura, T.; Miyagawa, N.; Tanaka, M.; Komiyama, A.; Kurata, S. Prevention of antibiotic-associated diarrhea in children by Clostridium butyricum MIYAIRI. Ped. Interl. 2003, 45, 86-90.

12. Ruszczyński, M.; Radzikowski, A.; Szajewska, H. Clinical trial: Effectiveness of Lactobacillus rhamnosus (strains E/ $\mathrm{N}$, Oxy and Pen) in the prevention of antibiotic-associated diarrhoea in children. Aliment. Pharmacol. Ther. 2008, 28, 154-161.

13. Wenus, C.; Goll, R.; Loken, E.B.; Biong, A.S.; Halvorsen, D.S.; Florholmen, J. Prevention of antibiotic-associated diarrhoea by a fermented probiotic milk drink. Eur. J. Clin. Nutr. 2008, 62, 299-301.

14. Allen, S.J.; Wareham, K.; Wang, D.; Bradley, C.; Sewell, B.; Hutchings, H.; Harris, W.; Dhar, A.; Brown, H.; Foden, A.; et al. A high-dose preparation of lactobacilli and bifidobacteria in the prevention of antibiotic-associated and Clostridium difficile diarrhoea in older people admitted to hospital: A multicentre, randomised, double-blind, placebo-controlled, parallel arm trial (PLACIDE). Health Technol. Assess. 2013, 17, 1-140.

15. Selinger, C.P.; Bell, A.; Cairns, A.; Lockett, M.; Sebastian, S.; Haslam, N. Probiotic VSL\#3 prevents antibiotic-associated diarrhoea in a double-blind, randomized, placebo-controlled clinical trial. J. Hosp. Infect. 2013, 84, 159-165.

16. Wullt, M.; Hagslatt, M.J.; Odenholt, I. Lactobacillus plantarum 299v for the treatment of recurrent Clostridium difficile-associated diarrhoea: A double-blind, placebo-controlled trial. Scand. J. Infect. Dis. 2003, 35, 365-367.

17. Surawicz, C.M.; McFarland, L.V.; Elmer, G.; Chinn, J. Treatment of recurrent Clostridium difficile colitis with vancomycin and Saccharomyces boulardii. Am. J. Gastroenterol. 1989, 84, 1285-1287. 
18. McFarland, L.V.; Surawicz, C.M.; Greenberg, R.N.; Elmer, G.W.; Moyer, K.A.; Melcher, S.A.; Bowen, K.E.; Cox, J.L. Prevention of $\beta$-lactam-associated diarrhea by Saccharomyces boulardii compared to placebo. Am. J. Gastroenterol. 1995, 90, 439-448.

19. Lewis, S.J.; Potts, L.F.; Barry, R.E. The lack of therapeutic effect of Saccharomyces boulardii in the prevention of antibiotic-related diarrhoea in elderly patients. J. Infect. 1998, 36, 171-174.

20. Duman, D.G.; Bor, S.; Ozütemiz, O.; Sahin, T.; Oğuz, D.; Iştan, F.; Vural, T.; Sandkci, M.; Işksal, F.; Simşek, I.; et al. Efficacy and safety of Saccharomyces boulardii in prevention of antibiotic-associated diarrhoea due to Helicobacterpylori eradication. Eur. J. Gastroenterol. Hepatol. 2005, 17, 1357-1361.

21. Kotowska, M.; Albrecht, P.; Szajewska, H. Saccharomyces boulardii in the prevention of antibiotic-associated diarrhoea in children: A randomized double-blind placebo-controlled trial. Aliment. Pharmacol. Ther. 2005, 21, 583-590.

22. Can, M.; Beşirbellioglu, B.A.; Avci, I.Y.; Beker, C.M.; Pahsa, A. Prophylactic Saccharomyces boulardii in the prevention of antibiotic-associated diarrhea: A prospective study. Med. Sci. Monit. 2006, 12, PI19-PI22.

23. Bravo, M.V.; Bunout, D.; Leiva, L.; de la Maza, M.P.; Barrera, G.; de la Maza, J.; Hirsch, S. Effect of probiotic Saccharomyces boulardii on prevention of AAD in adult outpatients with amoxicillin treatment. Rev. Med. Chile. 2008, 136, 981-988.

24. Pozzoni, P.; Riva, A.; Bellatorre, A.G.; Amigoni, M.; Redaelli, E.; Ronchetti, A.; Stefani, M.; Tironi, R.; Molteni, E.E.; Conte, D.; et al. Saccharomyces boulardii for the prevention of antibiotic-associated diarrhea in adult hospitalized patients: A single-center, randomized, double-blind, placebo-controlled trial. Am. J. Gastroenterol. 2012, 107, 922-931.

25. Shan, L.; Hou, P.; Wang, Z.; Liu, F.R.; Chen, N.; Shu, L.H.; Zhang, H.; Han, X.H.; Han, X.X.; Cai, X.X.; et al. Prevention and treatment of diarrhea with Saccharomyces boulardii in children with acute lower respiratory tract infections. Benef. Microbes 2013, 4, 329-334.

26. Arvola, T.; Laiho, K.; Torkkeli, S.; Mykkanen, H.; Salminen, S.; Maunula, L.; Isolauri, E. Prophylactic Lactobacillus GG reduces antibiotic-associated diarrhea in children with respiratory infections: A randomized study. Pediatrics 1999, 104, e64.

27. Thomas, M.R.; Litin, S.C.; Osmon, D.R.; Corr, A.P.; Weaver, A.L.; Lohse, C.M. Lack of effect of Lactobacillus GG on antibiotic-associated diarrhea: A randomized, placebo-controlled trial. Mayo Clin. Proc. 2001, 76, 883-889.

28. Miller, M.; Gravel, D.; Mulvey, M.; Taylor, G.; Gardam, M.; McGeer, A.; Hutchinson, J.; Moore, D.; Kelly, S.; Boyd, D.; et al. Health care-associated Clostridium difficile infection in Canada: Patient age and infecting strain type are highly predictive of severe outcome and mortality. Clin. Infect. Dis. 2010, 50, 194-201.

29. Morrow, L.E.; Kollef, M.H.; Casale, T.B. Probiotic prophylaxis of ventilator-associated pneumonia: A blinded, randomized, controlled trial. Am. J. Respir. Crit. Care Med. 2010, 182, 1058-1064.

30. Hickson, M.; D’Souza, A.L.; Muthu, N.; Rogers, T.R.; Want, S.; Rajkumar, C.; Bulpitt, C.J. Use of probiotic Lactobacillus preparation to prevent diarrhoea associated with antibiotics: Randomised double blind placebo controlled trial. Br. Med. J. 2007, 335, 80-83. 
31. Dietrich, C.G.; Kottmann, T.; Alavi, M. Commercially available probiotic drinks containing Lactobacillus casei DN-114001 reduce antibiotic-associated diarrhea. World J. Gastrol. 2014, 20, 15837-15844.

32. Plummer, S.; Weaver, M.A.; Harris, J.C.; Dee, P.; Hunter, J. Clostridium difficile pilot study: Effects of probiotic supplementation on the incidence of C. difficile diarrhoea. Int. Microbiol. 2004, 7, 59-62.

33. Rafiq, R. Prevention of Clostridium difficile ( $C$. difficile) diarrhea with probiotic in hospitalized patients treated with antibiotics. Gastroenterology 2007, 132, A187.

34. Stein, G.Y.; Nanim, R.; Karniel, E.; Moskowitz, I.; Zeidman, A. Probiotics as prophylactic agents against antibiotic-associated diarrhea in hospitalized patients. Harefuah 2007, 146, 520-522, 575.

35. Beausoleil, M.; Fortier, N.; Guénette, S.; L'ecuyer, A.; Savoie, M.; Franco, M.; Lachaine, J.; Weiss, K. Effect of a fermented milk combining Lactobacillus acidophilus C11285 and Lactobacillus casei in the prevention of antibiotic-associated diarrhea: A randomized, double-blind, placebo-controlled trial. Can. J. Gastroenterol. 2007, 21, 732-736.

36. Sampalis, J.; Psaradellis, E.; Rampakakis, E. Efficacy of BIO K+ CL1285 ${ }^{\circledR}$ in the reduction of antibiotic-associated diarrhea-A placebo controlled double-blind randomized, multi-center study. Arch. Med. Sci. 2010, 6, 56-64.

37. Gao, X.W.; Mubasher, M.; Fang, C.Y.; Reifer, C.; Miller, L.E. Dose-Response Efficacy of a Proprietary Probiotic Formula of Lactobacillus acidophilus CL1285 and Lactobacillus casei LBC80R for Antibiotic-Associated Diarrhea and Clostridium difficile-Associated Diarrhea Prophylaxis in Adult Patients. Am. J. Gastroenterol. 2010, 105, 1636-1641.

38. McFarland, L.V.; Surawicz, C.M.; Greenberg, R.N.; Fekety, R.; Elmer, G.W.; Moyer, K.A.; Melcher, S.A.; Bowen, K.E.; Cox, J.L.; Noorani, Z.; et al. A randomized placebo-controlled trial of Saccharomyces boulardii in combination with standard antibiotics for Clostridium difficile disease. JAMA 1994, 271, 1913-1918.

39. Surawicz, C.M.; McFarland, L.V.; Greenberg, R.N.; Fekety, R.; Elmer, G.W.; Moyer, K.A.; Melcher, S.A.; Bowen, K.E.; Cox, J.L.; Noorani, Z.; et al. The search for a better treatment for recurrent Clostridium difficile disease: Use of high-dose vancomycin combined with Saccharomyces boulardii. Clin. Infect. Dis. 2000, 31, 1012-1017.

40. Pochapin, M. The effect of probiotics on Clostridium difficile diarrhea. Am. J. Gastroenterol. 2000, 95, S11-S13.

41. Lawrence, S.J.; Korzenik, J.R.; Mundy, L.M. Probiotics for recurrent Clostridium difficile disease. J. Med. Microbiol. 2005, 54, 905-906.

42. Dinleyici, E.C.; Kara, A.; Ozen, M.; Vandenplas, Y. Saccharomyces boulardii CNCM I-745 in different clinical conditions. Expert Opin. Biol. Ther. 2014, 14, 1593-1609.

43. Auclair, J.; Frappier, M.; Millette, M. Lactobacillus acidophilus C1285, L. casei LBC80R and L. rhamnosus CLR2 (Bio-K+): Characterization, manufacture, mechanisms of action and quality control of a specific probiotic combination for primary prevention of Clostridium difficile infections. Clin. Infect. Dis. 2015, in press.

44. Guarner, F.; Khan, A.G.; Garisch, J.; Eliakim, R.; Gangl, A.; Thomson, A.; Krabshuis, J.; Lemair, T.; Kaufmann, P.; de Paula, J.A.; et al. World Gastroenterology Organisation Global Guidelines: Probiotics and prebiotics October 2011. J. Clin. Gastroenterol. 2012, 46, 468-481. 
45. Avadhani, A.; Miley, H. Probiotics for prevention of antibiotic-associated diarrhea and Clostridium difficile-associated disease in hospitalized adults-A meta-analysis. J. Am. Acad. Nurse Pract. 2011, 23, 269-274.

46. Goldenberg, J.Z.; Ma, S.S.; Saxton, J.D.; Martzen, M.R.; Vandvik, P.O.; Thorlund, K.; Guyatt, G.H.; Johnston, B.C. Probiotics for the prevention of Clostridium difficile-associated diarrhea in adults and children. Cochrane Database Syst. Rev. 2013, 5, CD006095.

47. Bermudez-Brito, M.; Plaza-Díaz, J.; Muñoz-Quezada, S.; Gómez-Llorente, C.; Gil, A. Probiotic mechanisms of action. Ann. Nutr. Metab. 2012, 61, 160-174.

48. Pattani, R.; Palda, V.A.; Hwang, S.W.; Shah, P.S. Probiotics for the prevention of antibiotic-associated diarrhea and $C$. difficile infection among hospitalized patients: Systematic review and meta-analysis. Open Med. 2013, 7, e56-e67.

49. McFarland, L.V.; Goh, S. Preventing Pediatric Antibiotic-Associated Diarrhea and Clostridium difficile Infections with Probiotics: A meta-analysis. World J. Meta-Anal. 2013, 1, 102-120.

50. Johnston, B.C.; Ma, S.S.; Goldenberg, J.Z.; Thorlund, K.; Vandvik, P.O.; Loeb, M.; Guyatt, G.H. Probiotics for the prevention of Clostridium difficile-associated diarrhea: A systematic review and meta-analysis. Ann. Intern. Med. 2012, 157, 878-888.

51. Liberati, A.; Altman, D.G.; Tetzlaff, J.; Mulrow, C.; Gøtzsche, P.C.; Ioannidis, J.P.; Clarke, M.; Devereaux, P.J.; Kleijnen, J.; Moher, D. The PRISMA statement for reporting systematic reviews and meta-analyses of studies that evaluate healthcare interventions: Explanation and elaboration. Br. Med. J. 2009, 339, b2700.

52. Egger, M.; Davey Smith, G.; Schneider, M.; Minder, C. Bias in meta-analysis detected by a simple, graphical test. Br. Med. J. 1997, 315, 629-634.

53. Moher, D.; Hopewell, S.; Schulz, K.F.; Montori, V.; Gøtzsche, P.C.; Devereaux, P.J.; Elbourne, D.; Egger, M.; Altman, D.G.; Consolidated Standards of Reporting Trials Group. CONSORT 2010 explanation and elaboration: Updated guidelines for reporting parallel group randomized trials. J. Clin. Epidemiol. 2010, 63, e1-e37.

54. Higgins, J.P.; Thompson, S.G.; Deeks, J.J.; Altman, D.G. Measuring inconsistency in meta-analyses. Br. Med. J. 2003, 327, 557-560.

55. Begg, C.B.; Mazumdar, M. Operating characteristics of a rank correlation test for publication bias. Biometrics 1994, 50, 1088-1101.

56. Peters, J.L.; Sutton, A.J.; Jones, D.R.; Abrams, K.R.; Rushton, L. Comparison of two methods to detect publication bias in meta-analysis. JAMA 2006, 295, 676-680.

57. McFarland, L.V.; Elmer, G.W.; Surawicz, C.M. Breaking the cycle: Treatment strategies for 163 cases of recurrent Clostridium difficile disease. Am. J. Gastroenterol. 2002, 97, 1769-1775.

58. McFarland, L.V. Systematic review and meta-analysis of Saccharomyces boulardii in adult patients. World J. Gastroenterol. 2010, 16, 2202-2222.

(C) 2015 by the authors; licensee MDPI, Basel, Switzerland. This article is an open access article distributed under the terms and conditions of the Creative Commons Attribution license (http://creativecommons.org/licenses/by/4.0/). 